Pecvnia, Monográfico (2008), pp. 185-203

\title{
Factores facilitadores e inhibidores del incremento del grado de diversificación en las empresas de Castilla y León
}

\author{
Almudena Martínez Campillo \\ amarc@unileon.es \\ Universidad de León \\ Organización de Empresas \\ Fac. de Ciencias Económicas y Empresariales \\ Campus de Vegazana, $\mathrm{s} / \mathrm{n}$ \\ 24071 León (España)
}

El presente trabajo estudia tres factores organizativos que pueden facilitar o inhibir el incremento del nivel de diversificación industrial: el tamaño corporativo, la diversificación internacional y la diversidad de producto inicial. En concreto, pretende analizar los efectos principales e interacción de dichos factores sobre la probabilidad de que las empresas aumenten el número de negocios donde participan. Para ello, tras revisar la literatura sobre diversificación y cambio estratégico, se plantean una serie de hipótesis, cuyo contraste se efectúa aplicando un análisis de regresión Logit. Los resultados indican que tanto el tamaño como la internacionalización de una empresa obstaculizan su crecimiento hacia nuevas líneas de actividad. Asimismo, este trabajo revela la existencia de
This study examines three organizational factors that may facilitate or inhibit the increase in industrial diversification: firm size, international diversification and initial product diversity. Specifically, the goal is to analyze their main and interaction effects on firms' propensity to enhance the number of business segments in which they operate. After revising literature on diversification and strategic change, the study proposes a number of hypotheses that are contrasted by applying a Logit regression. Results indicate that size and internationalization of the firm restrain significantly its growth toward new activities. Also, our findings reveal that there are significant interactions among both variables and the initial product diversity 
interacciones significativas entre ambas variables y la diversidad de producto inicial que influyen favorablemente en el incremento de la diversificación industrial.

Palabras clave: Tamaño corporativo, Diversificación internacional, Diversidad inicial, Diversificación industrial, Cambio estratégico, Logit. that affect positively on the increase in industrial diversification.

Key words: Firm size, Geographic diversification, Initial product diversity, Industrial diversification, Strategic change, Logit.

\section{INTRODUCCIÓN}

El contexto económico actual se caracteriza por ser incierto y turbulento. Durante las últimas décadas, la mayoría de los sectores industriales de los países desarrollados han sido objeto de profundas transformaciones en sus entornos. En este nuevo escenario de fuerte hostilidad ambiental, uno de los principales argumentos esgrimidos para explicar la supervivencia corporativa se encuentra fuertemente ligado al proceso de cambio estratégico experimentado por las empresas (Hambrick y D'Aveny 1988; Porter 1991; llinitch et al. 1996). En efecto, es ampliamente conocido que la globalización y el cambio tecnológico han motivado que las empresas rediseñen sus estrategias, de modo que sea factible responder a las amenazas y/o aprovechar las oportunidades que brinda el nuevo marco institucional y sectorial (Tsoukas y Chia 2002). De esta manera, el cambio estratégico emerge como uno de los temas de investigación más tratados por los académicos tanto nacionales como internacionales, con el fin de poder conocer con mayor profundidad los factores que afectan al mismo y su efecto en la supervivencia empresarial (Rajagopalan y Spreitzer 1997).

Si se revisa la literatura sobre los posibles determinantes del cambio estratégico, se advierte la existencia de dos posiciones teóricas antagónicas que han gozado de gran atención durante las dos últimas décadas (Zúñiga y Vicente 2006). En primer lugar, la corriente adaptativa, cuya premisa es que el cambio estratégico ocurre frecuentemente en las empresas, ya que a medida que las condiciones que rodean a la empresa se modifican, también deben hacerlo las estrategias empresariales (Smith y Grimm 1987). En segundo lugar, el enfoque ecológico, que defiende que las organizaciones son inertes, es decir, que la estrategia es una característica que se fija al comienzo de la vida corporativa y permanece relativamente constante a lo largo del tiempo (Freeman y Boeker 1984). 
El presente estudio se apoya en la primera corriente de estudio, que argumenta a favor del cambio estratégico y presume la existencia de ventajas asociadas al mismo. En concreto, se centra en la denominada perspectiva evolutiva adaptativa (Teece et al. 1997), que establece que las empresas pueden renovar sus estrategias cuando el entorno varía, pero que este cambio sucede lenta y gradualmente porque dentro de las organizaciones existen factores que pueden tanto promover como obstaculizar dicho cambio (Kelly y Amburgey 1991; Helfat 1998). A este respecto, la literatura señala que "cualquier estudio referente a un cambio estratégico se beneficiará de la consideración simultánea de los factores facilitadores e inhibidores de dicho cambio" (Zajac y Kraatz 1993: 85).

El interés de esta investigación radica en estudiar cómo afectan tres factores internos sobre una modalidad concreta de cambio estratégico: el incremento del grado de diversificación industrial. Específicamente, se trata de analizar los efectos principales e interacción del tamaño corporativo, la diversificación internacional y la diversidad de producto inicial sobre la propensión de las empresas a ampliar su campo de actividad.

El estudio contribuye a la literatura existente por las siguientes razones: Primero, porque integra argumentos de dos corrientes de investigación diferentes, la estrategia de diversificación y el cambio estratégico. Segundo, porque estudia un proceso de cambio estratégico muy relevante en el mundo empresarial y frecuentemente analizado -el incremento del nivel de diversificación industrial-, pero cuya evidencia empírica ha resultado inconsistente. Tercero, porque considera simultáneamente tres factores ampliamente estudiados en la literatura previa, que ofrecen potencial tanto para facilitar como para inhibir dicho proceso de cambio. Cuarto, porque analiza tanto los efectos principales como los efectos interacción de esos tres factores sobre la reorientación estratégica analizada.

El resto del trabajo ha sido estructurado como sigue: En la segunda sección se revisa la literatura sobre los posibles efectos facilitadores e inhibidores de los tres factores considerados, presentado las hipótesis que serán objeto de validación empírica. Las cuestiones metodológicas se abordan en la tercera sección. En la cuarta sección se exponen los resultados alcanzados. El trabajo termina recogiendo las conclusiones más relevantes del estudio realizado. 


\section{MARCO TEÓRICO E HIPÓTESIS}

El proceso de diversificación dentro de las empresas ha recibido mucha atención en la literatura durante las últimas décadas (Palich et al. 2000). Varias ramas del pensamiento económico y estratégico han incluido esta estrategia dentro de los principales argumentos de sus teorías, reconociendo su influencia sobre el crecimiento, resultado y ventaja competitiva de las empresas. En este sentido, el incremento del nivel de diversificación industrial puede ser considerado como un cambio estratégico esencial en el contexto actual, debido a las implicaciones que puede tener sobre el nivel de riesgo asumido, acceso a oportunidades, recursos y capacidades, eficiencia y efectividad de una empresa (Miller 2006). Las organizaciones tienden a competir en múltiples líneas de actividad poniendo de manifiesto una propensión hacia este tipo de procesos, de manera que, una vez desencadenado el crecimiento, deben seguir más de una dirección de desarrollo para garantizar su competitividad en los mercados (Sharma y Kesner 1996). Como consecuencia de ello, la diversificación industrial constituye uno de los fenómenos más característicos de la evolución del moderno capitalismo empresarial.

El objetivo de este trabajo es examinar el impacto de tres factores organizativos que pueden incidir sobre el incremento del nivel de diversificación industrial. Concretamente, se trata de analizar los efectos principales e interacción del tamaño corporativo, la diversificación internacional y la diversidad de producto inicial. Seguidamente se plantean dos hipótesis alternativas, derivadas del posible efecto facilitador e inhibidor de cada factor sobre la variable de interés.

\section{Tamaño corporativo}

La relación entre el tamaño corporativo y la capacidad para diversificar ha sido frecuentemente analizada, si bien las conclusiones han resultado controvertidas. Por un lado, el tamaño empresarial puede permitir que una compañía alcance economías de escala y alcance, así como facilitar el acceso a recursos denegados para empresas más pequeñas. Por tanto, puede ayudar a la empresa a la hora de afrontar riesgos, soportar contratiempos e iniciar cualquier tipo de cambio (Kimberly y Evanisko 1981; Haveman 1993; Zajac et al. 2000). Además, un tamaño empresarial importante proporciona un mayor poder de mercado para tratar con los "stakeholders", tanto en el entorno técnico como en el institucional (Pfeffer y Salancik 1978; Baum 1996). Consecuentemente, estos argumentos 
son consistentes con el efecto facilitador del tamaño en el incremento de la diversificación industrial, tal como concluyen algunos estudios previos (Merino y Rodríguez 1997; Silverman 1999; Villalonga 2004).

Por otro lado, un tamaño creciente puede inhibir la habilidad para el cambio en el interior de una organización. La evidencia ha demostrado que las empresas más grandes sufren un mayor grado de formalización y burocracia (Baum 1996; Haveman 1993), lo cual tiende a dificultar cualquier proceso de cambio dentro de las mismas. Asimismo, las grandes empresas suelen tener grupos diversos de "stakeholders" con intereses distintos entre sí, resultando más difícil lograr el consenso requerido para llevar cabo una reorientación estratégica (Kelly y Amburgey 1991; Stoeberl et al. 1998). Por tanto, la compleja estructura organizativa y la diversidad de intereses dentro de las empresas de gran tamaño pueden restringir su crecimiento hacia nuevos negocios.

hipótesis:

De acuerdo con lo argumentado, se establecen las siguientes

H1A: Existe una relación positiva entre el tamaño de la empresa y la probabilidad de incrementar el grado de diversificación industrial.

H1B: Existe una relación negativa entre el tamaño de la empresa y la probabilidad de incrementar el grado de diversificación industrial.

\section{Internacionalización}

Diversas investigaciones han examinado el efecto de la internacionalización en las empresas. Sin embargo, un número más escaso de estudios ha tratado de analizar su relación con la diversificación industrial (Tallman y Li 1996; Hitt et al. 1997; Bengtsson 2000; Geringer et al. 2000, entre otros). Dicha relación requiere atención como consecuencia del gran número de empresas que están adoptando una estrategia global (diversificación industrial+diversificación internacional), y teniendo en cuenta que ambas diversificaciones pueden estar asociadas positiva o negativamente. En primer lugar se va a considerar el escenario para una posible relación positiva. Las compañías que compiten en mercados globales probablemente tienen que enfrentar una mayor diversidad de necesidades y preferencias de sus clientes. Por tanto, necesitan invertir en nuevos productos para ser competitivas tanto a nivel internacional como nacional 
(Nachum 2004). Asimismo, dichas empresas pueden invertir en I+D y recuperar sus inversiones de un modo más rápido, como consecuencia de las sinergias resultantes de la demanda global existente para los productos que ofrecen (Kotabe 1990; Aw y Batra 1998). Otros beneficios adicionales pueden proceder de la estabilización de las ganancias o del aprovechamiento de las asimetrías de información (Buhner 1987). Por tanto, es probable que la diversificación internacional refuerce la necesidad y las capacidades requeridas para abordar la diversificación industrial a lo largo del tiempo. Acorde con ello, el estudio de Davies et al. (2001) concluye que las estrategias de diversificación industrial e internacional son, en general, complementarias.

El escenario opuesto implica que las empresas poseen recursos limitados para abordar ambas estrategias y deben elegir entre una de ellas (Sambharya 1995). En cualquier momento de tiempo, los diferentes proyectos de una empresa compiten por la captación de esos recursos, de manera que un tipo de diversificación puede ser sustituido por el otro. Según Chandler (1962), tradicionalmente las empresas se han implicado en estrategias de diversificación industrial antes de entrar en nuevos mercados geográficos extranjeros. Sin embargo, en la actualidad, las empresas pueden abordar estrategias de diversificación internacional con carácter previo a la diversificación industrial para conseguir ventajas de sus competencias distintivas especializadas. En este sentido, Bengtsson (2000) concluye que, durante el período comprendido entre 1985 y 1998, las empresas suecas experimentaron fuertes incrementos en el nivel de internacionalización y reducciones considerables en la diversificación industrial. Todo lo anterior implica la existencia de una relación negativa entre ambas variables. siguientes hipótesis:

Conforme a lo establecido previamente, se formulan las

H2A: Existe una relación positiva entre la diversificación internacional y la probabilidad de incrementar el grado de diversificación industrial.

H2B: Existe una relación negativa entre la diversificación internacional y la probabilidad de incrementar el grado de diversificación industrial. 


\section{Diversidad de producto inicial}

Poca investigación existe sobre el efecto de la diversidad de producto inicial en el nivel de diversificación posterior. Sin embargo, es posible asumir que un elevado nivel de diversidad inicial puede influir tanto positiva como negativamente sobre el incremento del número de negocios donde una empresa participa. Desde el punto de vista positivo, una gran diversidad inicial de producto representa la existencia de economías de alcance, ventajas derivadas del efecto experiencia, acceso a lógicas dominantes variadas y otros recursos y capacidades que, en definitiva, suponen una mayor oportunidad para aumentar la diversificación industrial.

Desde el punto de vista negativo, cabe señalar la posibilidad de que se originen rentabilidades decrecientes respecto a la diversificación. La aparición de deseconomías de escala, una necesidad superior de información y coordinación, y el aumento en los requisitos de procesamiento de la información pueden incrementar los costes burocráticos, anulando los efectos positivos de la diversificación y originando un freno al desarrollo de la empresa hacia nuevas líneas de actividad (Hill y Hansen 1991).

Acorde con dichos argumentos, se proponen sendas hipótesis:

H3A: Existe una relación positiva entre la diversidad de producto inicial y la probabilidad de incrementar el grado de diversificación industrial.

H3B: Existe una relación negativa entre la diversidad de producto inicial y la probabilidad de incrementar el grado de diversificación industrial.

En suma, cabe señalar que los tres factores considerados pueden proporcionar recursos y capacidades que faciliten la ampliación del campo de actividad de una empresa, o, alternativamente, incrementar los costes burocráticos, requisitos de procesamiento de información y determinadas deseconomías, restringiendo el incremento de la diversificación industrial. Por tanto, la influencia de estos tres factores puede depender, no sólo de los niveles propios de cada uno de ellos, sino también del nivel alcanzado por el resto. Así, es posible establecer las siguientes hipótesis respecto a las posibles interacciones existentes entre los factores objeto de estudio. 


\section{Efectos interacción}

Si tanto el tamaño corporativo como la diversidad de producto inicial pueden facilitar e inhibir el aumento del grado de diversificación industrial, cabe esperar que sus efectos combinados también tengan influencia sobre dicho proceso. Por un lado, es posible deducir que ambos factores pueden trabajar de forma sinérgica, proporcionando economías de escala y alcance, recursos y oportunidades, así como capacidades para obtener ventajas de la entrada en nuevas actividades. Por otro lado, también cabe la posibilidad de que cada factor puede reforzar los efectos negativos del otro. De esta manera, se pueden contrastar los efectos moderadores como sigue:

H4A: El tamaño de la empresa modera la relación positiva existente entre la diversidad de producto inicial y la probabilidad de incrementar el grado de diversificación industrial.

H4B: El tamaño de la empresa modera la relación negativa existente entre la diversidad de producto inicial y la probabilidad de experimentar un incremento en el grado de diversificación industrial.

Además, también es posible que el impacto de la internacionalización sobre el incremento de la diversificación industrial pueda depender de la dimensión de la empresa. Ello puede ser debido al efecto conjunto de las ventajas asociadas con el tamaño corporativo y la internacionalización. Alternativamente, también puede producirse el efecto inverso como consecuencia de los inconvenientes derivados de la dimensión de la empresa y de los costes burocráticos implicados en la diversificación internacional. Por tanto, cabe plantear las siguientes hipótesis:

H5A: El tamaño de la empresa modera la relación positiva existente entre la diversificación internacional y la probabilidad de incrementar el grado de diversificación industrial.

H5B: El tamaño de la empresa modera la relación negativa existente entre la diversificación internacional y la probabilidad de incrementar el grado de diversificación industrial. 
Por último, la diversificación internacional puede moderar la influencia de la diversidad de producto inicial, en la medida en que una empresa que compite con productos diversos puede tener mejor acceso a diferentes mercados. Alternativamente, es posible señalar que una empresa que ha seguido una diversificación industrial tiene que cumplir determinados requisitos burocráticos, de coordinación e información antes de que pueda emprender una diversificación internacional. Acorde con ello se proponen las siguientes hipótesis:

H6A: La diversificación internacional modera la relación positiva existente entre la diversidad de producto inicial y la probabilidad de incrementar el grado de diversificación industrial.

H6B: La diversificación internacional modera la relación negativa existente entre la diversidad de producto inicial y la probabilidad de incrementar el grado de diversificación industrial.

Figura 1: Modelo objeto de estudio

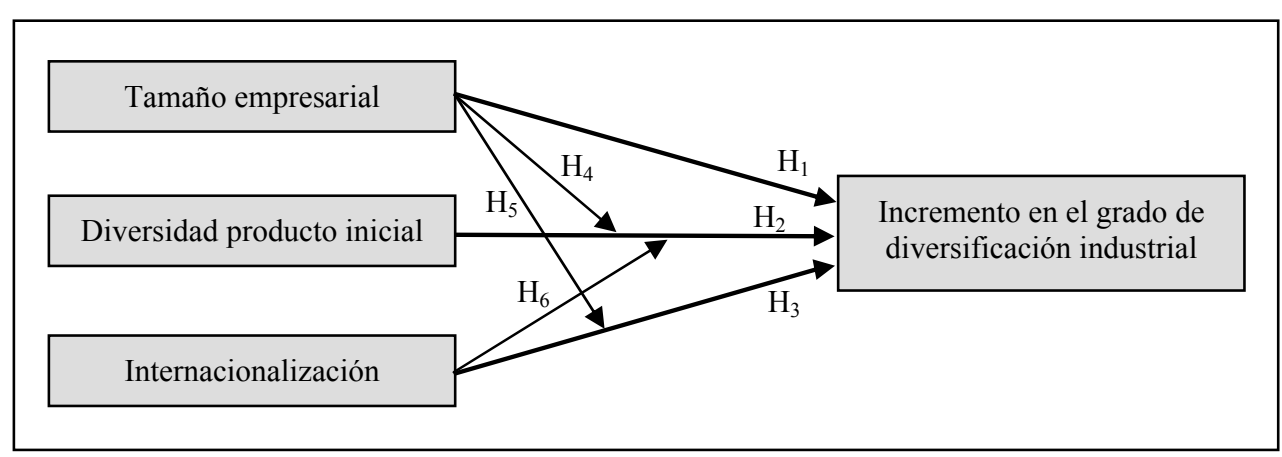

\section{METODOLOGÍA}

\subsection{Muestra y datos}

La población seleccionada para contrastar las hipótesis está integrada por todas las empresas industriales de Castilla y León durante el período comprendido entre 1990 y 2000. La muestra final queda compuesta por 104 empresas, que son las compañías que satisfacen dos condiciones: (1) permanecer activas en el mercado a lo largo de todo el periodo 
analizado, y (2) disponer de datos para medir todas las variables de interés en dicho período. El error muestral generado fue del 8,5\% con un nivel de confianza del $95 \%$. La información requerida para obtener esta muestra se obtuvo a partir de la Guía Empresarial de Castilla y León y las bases de datos Duns\&Bradstreet y SABI (Sistema de Análisis de Balances Ibéricos).

\subsection{Variable dependiente}

La variable dependiente del estudio recoge la probabilidad de que una empresa haya experimentado un incremento en el grado de diversificación industrial a lo largo del período 1990-2000. Por tanto, se trata de una variable dicotómica que toma el valor de 1 en caso de haberse producido dicho cambio estratégico, y el valor de 0 en caso contrario.

El grado de diversificación industrial fue medido utilizando un índice de recuento de negocios, siendo éstos determinados a partir del sistema de códigos SIC a nivel de cuatro dígitos (Bowen y Wiersema 2005). Dicha elección radica en la sencillez y objetividad que ofrece este procedimiento, ya que se encuentra vinculado a clasificaciones oficiales válidas para todos los investigadores. La fuente de información sobre los distintos negocios a nivel de cuatro dígitos SIC de cada empresa en cada período (1990 y 2000) fue el directorio Duns\&Bradstreet.

A partir de ahí, el incremento del grado de diversificación industrial se cuantificó utilizando la medida adoptada por Hill y Hansen (1991), que consiste en dividir el grado de diversificación del período posterior (2000) entre aquel correspondiente al período anterior (1990). Cuando el valor resultante supera la unidad se considera que la empresa ha experimentado un crecimiento en el número de líneas de actividad donde participa, mientras que si el valor es igual o inferior a uno cabe deducir el mantenimiento o la reducción del grado de diversificación industrial, respectivamente.

\subsection{Variables independientes}

Las variables independientes incluidas en el modelo son los tres factores organizativos con potencial tanto para favorecer como para limitar un proceso de incremento en el grado de diversificación industrial, esto es, el tamaño corporativo, la diversificación internacional y la diversificad de producto inicial. 
- El tamaño corporativo fue medido a través del logaritmo del número total de empleados de la empresa. En concreto, se calculó el valor medio anual de tal indicador para el periodo 1990-1999.

- La diversificación internacional es una variable dicotómica que refleja si la empresa abordó esta opción estratégica de crecimiento durante el período 1990-1999, adoptando un valor igual a 1 en caso afirmativo e igual a 0 en otro caso.

- La diversidad de producto inicial fue medida a través de una variable cuantitativa que recogía el grado de diversificación industrial de la empresa en el año 1990, es decir, el número de negocios diferentes a nivel de cuatro dígitos SIC en los que la empresa desarrolló su actividad en dicho ejercicio.

Toda la información requerida para calcular estas tres variables se tomó del Directorio Duns\&Bradstreet y de la base de datos SABI.

\subsection{Especificación del modelo}

La técnica estadística multivariante que permite estimar la probabilidad de que ocurra un suceso es el modelo de regresión Logit. La idoneidad de esta técnica se deriva de la naturaleza de la variable dependiente, categórica y dicotómica, y de las independientes, donde se combinan variables continuas con categóricas. Además, la regresión Logit resulta adecuada cuando se desconocen las condiciones de normalidad de las variables y no se plantean restricciones respecto a la igualdad de la matriz de varianzas-covarianzas (Hoetker 2007). El tamaño de la muestra y la ausencia de altos niveles de multicolinealidad permiten aplicar con garantías dicha técnica. Específicamente, la ecuación de regresión Logit quedó definida del siguiente modo:

$\operatorname{Prob}(Y=1)=\operatorname{Prob}($ Incrementos en diversificación industrial $)=e^{\mathrm{z}} / 1+e^{\mathrm{z}}=1 /\left(1+e^{-\mathrm{z}}\right)$

donde $Z=\alpha+\beta 1$ Tamaño $+\beta 2$ Internacionalización $+\beta 3$ Diversidad inicial $+\beta 4$ Tamaño $\times$ Internacionalización $+\beta 5$ Tamaño $\times$ Diversidad inicial $+\beta 6$ Internacionalización $\times$ Diversidad inicial 


\section{RESULTADOS}

La Tabla 1 presenta los principales estadísticos descriptivos (media y desviación típica) de las variables del estudio, así como los coeficientes de correlación entre ellas. Tras observar dichos coeficientes, es posible deducir que las variables independientes se encuentran débilmente interrelacionadas entre sí.

Tabla 1: Estadísticos descriptivos y matriz de correlaciones

\begin{tabular}{|l|c|c|c|c|c|c|}
\hline Variables & Media & S.D. & $\mathbf{1}$ & $\mathbf{2}$ & $\mathbf{3}$ & $\mathbf{4}$ \\
\hline 1. $\Delta$ Diversificación industrial & 0,35 & 0,23 & 1,00 & & & \\
\hline 2. Tamaño corporativo & 2,55 & 0,97 & $-0,04$ & 1,00 & & \\
\hline 3. Internacionalización & 0,53 & 0,18 & $-0,12$ & 0,17 & 1,00 & \\
\hline 4. Diversidad inicial & 2,70 & 1,88 & 0,01 & 0,11 & 0,09 & 1,00 \\
\hline
\end{tabular}

La Tabla 2 recoge los resultados obtenidos tras efectuar la regresión logística, así como la bondad del ajuste y la capacidad predictiva del modelo. El programa estadístico SPSS posee varios métodos de selección que permiten considerar exclusivamente aquellas variables independientes que explican la variable dependiente. Por esta razón, la tabla sólo muestra las variables que han resultado significativas para discriminar entre las empresas que experimentaron incrementos en el grado de diversificación industrial y las que no.

Tabla 2: Estimación del modelo de regresión logística

LOGIT // Variable Dependiente: PROBABILIDAD DE INCREMENTAR LA DIVERSIFICACIÓN INDUSTRIAL $\mathrm{N}^{\circ}$ total de observaciones: 104

$\mathrm{N}^{\mathrm{o}}$ de observaciones con variable dependiente 1:37

$\mathrm{N}^{\mathrm{o}}$ de observaciones con variable dependiente $0: 67$

\begin{tabular}{|l|c|c|c|c|}
\hline Variables independientes & $\boldsymbol{\beta}$ & E.T. & Wald & $\operatorname{Exp}(\boldsymbol{\beta})$ \\
\hline Tamaño corporativo & $-0,007 \dagger$ & 0,005 & 2,398 & 0,993 \\
\hline Internacionalización & $-3,126 * *$ & 1,028 & 9,243 & 0,044 \\
\hline Diversidad inicial $\times$ Tamaño corporativo & $0,005 \dagger$ & 0,003 & 2,924 & 1,005 \\
\hline Diversidad inicial $\times$ Internacionalización & $1,908 * * *$ & 0,055 & 12,159 & 6,740 \\
\hline Constante & $-0,470$ & 0,380 & 1,526 & 0,625 \\
\hline $\begin{array}{l}\text { Chi-cuadrado: } 29,370^{* * *} \\
\mathrm{R}^{2} \text { Nagelkerke: } 24,7 \% \\
\% \text { correctamente clasificado: } 75,2\end{array}$ & & & \\
\hline
\end{tabular}

Las variables continuas que forman los términos interacción fueron centradas para evitar problemas de multicolinealidad. Dicha transformación no afecta al resultado.

$\dagger p<0,10 ; * p<0,05 ; * * p<0,01 ; * * * p<0,001$ 
A la vista de estos resultados, es posible afirmar que cuatro variables explicativas; en concreto, el tamaño corporativo, la diversificación internacional, la interacción entre el tamaño corporativo y la diversidad de producto inicial, y la interacción entre la diversificación internacional y la diversidad de producto inicial, han resultado significativas para explicar la probabilidad de que una empresa aumente su ámbito de actuación. En concreto, la ecuación de regresión logística queda expresada como sigue:

$\operatorname{Prob}(Y=1)=\operatorname{Prob}($ Incrementos en diversificación industrial $)=e^{\mathrm{z}} / 1+e^{\mathrm{z}}=1 /\left(1+e^{-\mathrm{z}}\right)$

donde $Z=-0,470-0,007$ Tamaño $-3,126$ Internacionalización $+0,005$ Tamaño $\times$ Diversidad inicial $+1,908$ Internacionalización $\times$ Diversidad inicial

Respecto a los efectos principales de los factores considerados, cabe deducir que las variables relativas al tamaño corporativo y la diversificación internacional resultaron negativas y significativas, lo cual indica que ambas variables por sí mismas ejercen un efecto inhibidor sobre la probabilidad de que una empresa incremente su nivel de diversificación industrial. Por tanto, estos resultados suponen validar las hipótesis $1 \mathrm{~B}$ y $2 \mathrm{~B}$, respectivamente. Además, parece interesante señalar que el efecto inhibidor del tamaño es mayor que el ejercido por la diversificación internacional. Los resultados alcanzados, sin embargo, no permiten demostrar la influencia de la diversidad de producto inicial sobre el cambio estratégico analizado.

Con relación a los efectos moderadores, señalar que resultaron positivas y significativas las dos interacciones siguientes: la existente entre el tamaño corporativo y la diversidad de producto inicial y la que relaciona a esta última con la diversificación internacional, siendo esta variable interacción las más significativa del modelo, y por tanto, la que más afecta a la hora de explicar la probabilidad de que una empresa decida ampliar su campo de actividad. Ello implica la aceptación de las hipótesis $4 \mathrm{~A}$ y $6 \mathrm{~A}$ respectivamente. Por otro lado, no se encontró ningún tipo de soporte para validar el efecto moderador del tamaño corporativo sobre la relación entre la diversificación internacional y el proceso de cambio estudiado.

De cara a valorar la significación global del modelo, el programa proporciona un valor del estadístico Chi-cuadrado para el conjunto de variables incluidas en la ecuación final igual a 29,370, con un 
nivel de significación igual a $p=0,000$. Este resultado indica que las variables independientes introducidas en el modelo son útiles para explicar el crecimiento del número de negocios dónde compiten las empresas. Por lo que respecta a la eficacia predictiva del modelo, cabe señalar que el $75,2 \%$ de las empresas de la muestra queda bien clasificado con la ecuación logística estimada.

\section{CONCLUSIONES}

El objetivo de esta investigación era estudiar, bajo la perspectiva evolutiva adaptativa, los efectos principales e interacción de tres factores organizativos con potencial para facilitar e inhibir el incremento de la diversificación industrial: el tamaño corporativo, la diversificación internacional y la diversidad de producto inicial. A partir de datos relativos una muestra de 104 empresas de Castilla y León para el período 1990-2000, se aplicó un análisis de regresión Logit, cuyos resultados soportaron algunos de los efectos objeto de estudio. Como conclusiones más relevantes del estudio se pueden destacar las siguientes.

A la vista de un análisis preliminar, es posible inferir que, inversamente a las predicciones del enfoque ecológico, y en consonancia con los principios de la perspectiva evolutiva adaptativa, un número considerable de empresas de la muestra (en concreto 37, es decir, un 35\% del total) decide cambiar su orientación estratégica e incrementar su nivel de diversificación industrial durante el período 1990-2000.

Respecto a los factores que influyen directamente en tal proceso de cambio, se ha verificado que tanto el tamaño corporativo como el seguimiento de una diversificación internacional obstaculizan significativamente el crecimiento del número de negocios en que una empresa compite. Por tanto, es posible deducir que un gran tamaño empresarial puede dificultar la capacidad de una compañía para incrementar el nivel de diversificación industrial, como consecuencia de que las grandes empresas suelen disponer de estructuras organizativas muy formalizadas, burocráticas e inflexibles que reducen la posibilidad de emprender cualquier proceso de reorientación estratégica. Por otro lado, dado que las empresas poseen recursos limitados, sus diferentes procesos de diversificación industrial e internacional parecen competir por la captación de tales recursos, de manera que un tipo de diversificación resulta sustituido por 
el otro. Este resultado coincide, por tanto, con los obtenidos por Bengtsson (2000) respecto a una muestra de empresas suecas.

No obstante, y sorprendentemente, cuando ambos factores (tamaño e internacionalización) se combinan con la diversidad de producto inicial, se originan efectos favorables sobre el incremento de la diversificación industrial. Por tanto, aunque la diversidad inicial de producto per se no parece impactar sobre el proceso de cambio analizado, sin embargo, puede resultar esencial para que la internacionalización de la empresa y su dimensión, que por sí solas ejercen un efecto inhibidor, puedan conllevar el desarrollo de la empresa hacia nuevas líneas de actividad. En efecto, esta investigación revela que las empresas con presencia en mercados internacionales y altos niveles de diversidad de producto inicial tienen posibilidad de ampliar su campo de actividad, ya que pueden acceder más fácilmente a nuevos mercados. Específicamente, la combinación de ambos factores parece ser el determinante más influyente a la hora de extender el ámbito de actuación de una empresa. Además, los resultados también indican que el tamaño corporativo y la diversidad de producto inicial actúan sinérgicamente, logrando economías de escala y alcance, recursos y capacidades que permiten obtener ventajas del incremento de la diversificación industrial.

En suma, los resultados de este trabajo ilustran la conveniencia de analizar los determinantes de una dimensión concreta del cambio estratégico; en este caso, el incremento del nivel de diversificación industrial, frente a la consideración de una variable genérica del mismo, tal como se ha hecho en la mayoría de los estudios previos. No obstante, cuando se examinan los efectos principales e interacción de dichos determinantes, la existencia de efectos moderadores significativos supone la necesidad de interpretar cautelosamente los efectos principales de los factores implicados.

Los hallazgos del estudio deberían ser considerados con prudencia debido a las siguientes limitaciones. Primero, la muestra utilizada incluye todas las empresas industriales de Castilla y León para las que existían datos sobre las variables objeto de estudio. Por tanto, representa una muestra de conveniencia. Segundo, el modelo de regresión debería ser estimado en otros contextos geográficos y considerando diferentes periodos de tiempo a fin de afianzar su validez. Tercero, el estudio examina la relación entre el incremento del grado de diversificación industrial y tres factores organizativos ampliamente analizados. Podría resultar interesante ampliar el modelo con otros posibles factores determinantes 
de dicho cambio estratégico (factores del entorno, características directivas, otros factores organizativos tales como la edad de la empresa o su complejidad estructural, etc.). Quinto, la aproximación utilizada para medir algunas variables puede resultar menos precisa de lo deseable.

Las limitaciones anteriores pueden justificar la existencia de hipótesis no validadas, lo cual supone un estímulo para seguir investigando al respecto. Además, extendiendo el camino iniciado con este estudio, como futura línea de investigación se plantea el análisis de las consecuencias del cambio estratégico analizado en la supervivencia de las empresas que lo abordan. Del mismo modo, también podría resultar interesante comparar los resultados generados por estas empresas con aquellos alcanzados por las compañías que deciden mantener o reducir su campo de actividad.

\section{BIBLIOGRAFÍA}

AW, B. \& G. BATRA (1998) "Firm Size and the Pattern of Diversification", International Journal of Industrial Organization, Vol. 16, No. 3, pp. 313-331.

BAUM, J.A.C. (1996) "Organizational Ecology". S. CleGG, C. HARDY \& W. NORD (eds.) Handbook of Organization Studies. London: Sage.

BENGTSSON, L. (2000) "Corporate Strategy in a Small Open Economy: Reducing Product Diversification while Increasing International Diversification", European Management Journal, Vol. 18, No. 4, pp. 444-453.

BoWen, H.P. \& M.F. Wiersema (2005) "Foreign-Based Competition and Corporate Diversification Strategy", Strategic Management Journal, Vol. 26, No. 12, pp. 1153-1171.

BUHNER, R. (1987) "Assessing International Diversification of West German Corporations", Strategic Management Journal, Vol. 8, No. 1, pp. 25-37.

CHANDLER, A. (1962) Strategy and Structure. Cambridge, MA: MIT Press.

DAVIES, S.W.; L. RonDI \& A. SEMBENELLI (2001) "Are Multinationality and Diversification Complementary or Substitute Strategies? An Empirical Analysis on European Leading Firms", International Journal of Industrial Organization, Vol. 19, No. 8, pp. 1315-1346.

Freeman, J. \& W. Boeker (1984) "The Ecologycal Analysis of Business Strategy", California Management Review, Vol. 23, No. 3, pp. 73-86. 
GeRINGER, M.J.; S. TALLMAN \& D.M. OLSEN (2000) "Product and International Diversification among Japanese Multinational Firms", Strategic Management Journal, Vol. 21, No. 1, pp. 51-80.

HAMBRICK, D.C. \& R.A. D'AVENI (1988) "Large Corporate Failures as Downward Spirals", Administrative Science Quarterly, Vol. 33, No. 1, pp. 1-23.

HAVEMAN, H.A. (1993) "Organizational Size and Change: Diversification in the Savings and Loan Industry after Deregulation", Administrative Science Quarterly, Vol. 38, No. 1, pp. 20-50.

Helfat, C.E. (1998) "Simple Indicators of Adaptation versus Rigidity in History-Dependent Firm Activities and Decision Rules", Industrial and Corporate Change, Vol. 7, No. 1, pp. 49-75.

Hill, C.W.L. \& G.S. HANSEN (1991) "A Longitudinal Study of the Cause and Consequences of Changes in Diversification in the U.S. Pharmaceutical Industry 1977-1986", Strategic Management Journal, Vol. 12, No. 3, pp. 187-199.

HITT, M.A.; R.E. HoSKISSON \& H. KIM (1997) "International Diversification: Effects on Innovation and Firm Performance in Product-Diversified Firms", Academy of Management Journal, Vol. 40, No. 4, pp. 767-798.

Hottker, G. (2007) "The Use of Probit and Logit Models in Strategic Management Research: Critical. Issues", Strategic Management Journal, Vol. 28, No. 4, pp. 331-343.

ILINITCH, A.Y.; R.A. D'AVENI \& A.Y. LEWIN (1996) "New Organisational Forms and Strategies for Managing in Hyper Competitive Environments", Organisation Science, Vol. 7, No. 3, pp. 211-220.

KeLLY, D. \& T.L. AMBURGEY (1991) "Organizational Inertia and Momentum: A Dynamic Model of Strategic Change", Academy of Management Journal, Vol. 34, No. 3, pp. 591-612.

KIMBERLY, J.R. \& M.J. EVANISKO (1981) "Organizational Innovation: The Influence of Individual, Organizational, and Contextual Factors on Hospital Adoption of Technological and Administrative Innovations", Academy of Management Journal, Vol. 24, No. 4, pp. 689-713.

KotABE, M. (1990) "The Relationship between Offshore Sourcing and Innovativeness of U.S. Multinational Firms: An Empirical Investigation", Journal of International Business Studies, Vol. 21, No. 4, pp. 623-638.

MERINO, F. \& D.R. RODRíGUEZ (1997) "A Consistent Analysis of Diversification Decisions with Non-Observable Firm Effects", Strategic Management Journal, Vol. 18, No. 9, pp. 733-743. 
MILLER, D.J. (2006) "Technological Diversity, Related Diversification and Firm Performance", Strategic Management Journal, Vol. 27, No. 7, pp. 601619.

NACHUM, L. (2004) "Geographic and Industrial Diversification of Developing Country Firms", Journal of Management Studies, Vol. 41, No. 2, pp. 273-294.

Palich, L.E.; L.B. Cardinal \& C.C. Miller (2000) "Curvilinearity in the Diversification-Performance Linkage: An Examination of Over Three Decades of Research", Strategic Management Journal, Vol. 21, No. 2, pp. 155-174.

Pfeffer, J. \& G. SAlaCIK (1978) The External Control of Organizations: A Resource Dependence Perspective. New York: Harper y Row.

PORTER, M.E. (1991) "Toward a Dynamic Theory of Strategy", Strategic Management Journal, Vol. 12, Special Issue, pp. 95-117.

Rajagopalan, N. \& G.M. Spreitzer (1997) "Toward a Theory of Strategic Change: A Multi-Lens Perspective and Integrative Framework", Academy of Management Review, Vol. 22, No. 1, pp. 48-79.

SAmbHARYA, M.B. (1995) "The Combined Effects of International Diversification and Product Diversification Strategies on the Performance of U.S. Based Multinational Corporations", Management International Review, Vol. 35, pp. 197-218.

Sharma, A. \& I.F. KeSNER (1996) "Diversifying Entry: Some ex ante Explanations for Postentry Survival and Growth", Academy of Management Journal, Vol. 39, No. 3, pp. 635-677.

SILVERMAN, B.S. (1999) "Technological Resources and the Direction of Corporate Diversification: Toward and Integration of the ResourceBased View and Transaction Cost Economics", Management Science, Vol. 45, No. 8, pp. 1109-1124.

SMITH, K.G. \& C.M. GRIMM (1987) "Environmental Variation, Strategic Change and Firm Performance: A Study of Railroad Deregulation", Strategic Management Journal, Vol. 8, No. 4, pp. 363-376.

StOeberL, P.A.; G.E. PARKer \& S. JoO (1998) "Relationship between Organizational Change and Failure in the Wine Industry: An Event History Analysis", Journal of Management Studies, Vol. 35, No. 4, pp. 537-555.

TALLmAn, S. \& J.T. LI (1996) "The Effects of International Diversity and Product Diversity on the Performance of Multinational Firms", Academy of Management Journal, Vol. 39, No. 1, pp. 179-196. 
TeECE, D.J.; G. PISANO \& A. Shuen (1997) "Dynamic Capabilities and Strategic Management", Strategic Management Journal, Vol. 18, No. 7, pp. 509533.

TSOUKAS, H. \& R. CHIA (2002) "On Organizational Becoming: Rethinking Organizational Change", Organization Science, Vol. 13, No. 5, pp. 567582.

VILlaLongA, B. (2004) "Does Diversification Cause the 'Diversification Discount'?", Financial Management, Vol. 33, No. 2, pp. 5-27.

ZAJAC, E.J. \& M.S. KRAATZ (1993) "A Diametric Forces Model of Strategic Change: Assessing the Antecedents and Consequences of Restructuring in the Higher Educational Industry", Strategic Management Journal, Vol. 14, Special Issue, pp. 83-102.

-, - \& R.K.F. BRESSER (2000) "Modelling the Dynamics of Strategic Fit: A Normative Approach to Strategic Change", Strategic Management Journal, Vol. 21, No. 4, pp. 429-453.

ZÚÑIGA, J.A. \& J.D. VICENTE (2006) "Strategic Moves and Organizational Survival in Turbulent Environments: The Case of Spanish Banks (19831997)" , Journal of Management Studies, Vol. 43, No. 3, pp. 485-519. 